Erwin J. O. Kompanje

\title{
Should we discharge comatose patients from intensive care to die in their own bed at home after withdrawal of mechanical ventilation?
}

Received: 23 February 2009

Accepted: 23 February 2009

Published online: 12 March 2009

(C) Springer-Verlag 2009

This editorial refers to the article available at: doi:10.1007/s00134-009-1452-1.

\section{E. J. O. Kompanje (}

Department of Intensive Care,

Erasmus MC University Medical Center Rotterdam,

P.O. Box 2040, 3000 CA, Rotterdam,

The Netherlands

e-mail: erwinkompanje@mac.com

In an intensive care unit (ICU), mechanical ventilation (MV) and vasopressive agents (VA) are commonly used life-sustaining treatments. Sometimes it is decided to withdraw these treatments for reasons of futility, disproportion or at the request of the patient, in almost all cases resulting in death of the patient within minutes or hours. There are significant differences among countries, resulting in percentages of withdrawal of mechanical ventilation of between 1 and $10 \%$ of all ICU admissions [1-4]. The time until death seems mostly dependent on the severity of illness and organ failure and on which treatments are withdrawn. After withdrawal of life-sustaining treatment, possibly distressing symptoms can occur, and these should be prevented and suppressed with opioids, sedatives and other medicaments. Most patients in whom life-sustaining intensive care measures are withdrawn die in the ICU (almost 90\%) or shortly after discharge from the ICU on a hospital ward (approximately $10 \%$ ). There are only a few publications mentioning discharge of patients to home directly from the ICU in order to die [5-10]. In this issue of Intensive Care Medicine, Huang et al. [11] report their experiences from an ICU in Taiwan. The authors did a retrospective observational study in a tertiary medical center. The patients were transported from the ICU to their homes on ventilator support. Blood pressure was maintained with inotropic drugs, and sedatives and opioids were administered for comfort. After arrival at the patient's home, the patient was placed in a bed in the main hall of the house, after which mechanical ventilation was withdrawn, the endotracheal tube removed and administration of vasoactive drugs stopped. Sedatives and opiods were continued. Death occurred in minutes to hours. Between 2003 and 2007, 969 of the patients admitted to the authors' ICU died, 346 (36\%) of them after being discharged to the patient's own home, where mechanical ventilation was withdrawn.

According to the sparse literature, this practice is remarkable from the Western perspective. For example, Steinhauser et al. [12] determined the factors considered important at the end of life by patients, their families, and physicians and other care providers. They measured the main rank scores for nine preselected attributes (freedom of pain; at peace with God; presence of family, mentally aware; treatment choices followed, finances in order; feel life was meaningful; resolve conflicts and die at home), with 1 being most important and 9 being least important. All groups ranked 'die at home' the least important and 'free of pain' the most important. An analysis exploring the quality of the dying experience on the ICU showed that family members of dying patients also rank pain control as the most important aspect for quality of dying on the ICU [13]. On the other hand, when asked directly, most people would prefer to die at home in their own familiar environment. Especially patients with terminal cancer or other chronic diseases, between a half and three quarters of whom would ideally choose a death at home, under a quarter actually die there. Most of them die in the hospital.

But most of these patients with terminal malignant diseases are completely conscious and would experience being at home, which is not the case with most of the 
patients in whom treatment is withdrawn for the reason of futility. Most of the patients are comatose or deeply sedated. The two patients described by Beuks et al. [5] were also conscious patients, and they were not mechanically ventilated. This in contrast with the patients described by Huang et al. In addition, Kallel et al. [6] and Boussarsar and Bouchoucha [7] also described how comatose ventilated patients are transported to their homes to die.

It is difficult to see how it can be in the best interests of an unconscious or sedated patient to be transported for the purpose of dying at their home. Most of the patients concerned are very sick, with severe organ failure, and most will not be able to experience their dying or transport home.

Transporting patients on ventilatory support and intravenous vasoactive medication can only be done with a physician-staffed mobile intensive care unit (MICU), which is a more expensive form of transportation than a normal ambulance. In many countries, ICUs have to provide their own transport team, taking intensive care physicians and intensive care nurses of the ICU. Consequently, insurance companies or ICU managers may judge that they cannot afford to make the investments needed in specialist transportation to allow the choice of a wellsupported death at home. On the other hand, it fits exactly within the context of a highly praised Dutch initiative: 'Stichting Ambulance Wens' ('Ambulance Wish Foundation,' http://www.ambulancewens.nl), which fulfills the wishes of terminal patients by transporting them with an ambulance to visit a particular place for the last time.

For some groups of patients, dying at home is of great importance. For example, Muslims have a specific perception of death and dying, and the majority of them desire to die at home in their own bed, surrounded by their family and friends $[6,7,14,15]$. Making death clinical and remote in a hospital setting (e.g., an ICU) is not in keeping with the Islamic tradition [14]. For these cultural or religious groups of patients, dying at home after withdrawal of mechanical ventilation or other intensive care measures could have substantial meaning.

For many patients originating from Western countries, dying at home after discharge from the ICU is not a notable issue. But as many ICUs in Western countries admit patients from many different cultures and religious beliefs, the service as described by Huang et al. is worth considering in providing the most appropriate care in pluralistic societies.

\section{References}

1. Ferrard E, Robert R, Ingrand $P$, Lemaire F (2001) Withholding and withdrawal of life support in intensive-care units in France: a prospective survey. Lancet 357:9-14

2. Lee DKP, Swinburne AJ, Fedullo AJ, Wahl GW (1994) Withdrawing care experience in a medical intensive care unit. JAMA 271:1358-1361

3. Nolin T, Andersson R (2003) Withdrawal of medical treatment in the ICU. A cohort study of 318 cases during 1994-2000. Acta Anaesthesiol Scand 47:501-507

4. Smedira NG, Evans BH, Grais LS, Cohen NH, Lo B, Cooke M, Schecter WP, Fink C, Epstein-Jaffe E, May C et al (1990) Withholding and withdrawal of life support from the critically ill. NEJM 322:309-315

5. Beuks BC, Nijhof AC, Meertens JH, Ligtenberg JJ, Tulleken JE, Zijlstra JG (2006) A good death. Intensive Care Med 32:752-753
6. Kallel H, Dammak H, Bahloul M, Ben Hamida C, Chelly H, Rekik N, Bouaziz M (2006) A good death: another break in the wall. Intensive Care Med 32:1915-1916

7. Boussarar M, Bouchoucha S (2006) Dying at home: cultural and religious preferences. Intensive Care Med 32:1917-1918

8. Mann S, Galler D, Williams P, Frost P (2004) Craing for patients and families at the end of life: withdrawal of intensive care in the patients home. $\mathrm{N} \mathrm{Z}$ Med J 117:U935

9. Ryder-Lewis M (2005) Going home from the ICU to die: a celebration of life. Nurs Crit Care 10:116-121

10. Hawdon JM, Williams S, Weindling AM (1994) Withdrawal of neonatal intensive care in the home. Arch Dis Childhood 71:F142-F144

11. Huang YC, Huang SJ, Ko WJ (2009) Going home to die from surgical intensive care units. Intensive Care Med. doi:10.1007/s00134-009-1452-1
12. Steinhauser KE, Christakis NA, Clipp EC, McNeilly M, McIntyre L, Tulsky JA (2000) Factors considered important at the end of life by patients, family, physicians and other care providers. JAMA 284:2476-2482

13. Mularski RA, Heine CE, Osborne ML, Ganzini L, Curtis JR (2005) Quality of dying in the ICU. Ratings by family members. Chest 128:280-287

14. Sheikh A (1998) Death and dying-a Muslim perspective. J Roy Soc Med 91:138-140

15. Gatrad AR (1994) Muslim customs surrounding death, bereavement, postmortem examinations, and organ transplants. BMJ 309:521-523 